\title{
The Barcode of Life Initiative: synopsis and prospective societal impacts of DNA barcoding of Fish
}

\author{
FILIPE O. COSTA AND GARY R. CARVALHO ${ }^{1}$
}

\begin{abstract}
Almost 250 years after the publication of the taxonomy-founding work Systema Naturae, by Carl Linnaeus, the inventory and catalogue of the planet's biodiversity is still far from complete: only $c a 1.5$ to 1.8 million of an estimated $10+$ million species are so far described. Notwithstanding the remarkable merits of the Linnean system, the task is too vast ever to be completed using current conventional approaches. Such a staggering reality, and the customary difficulty that the scientific community and society in general experience to access taxonomic knowledge, has prompted the search for novel tools or approaches for species identification. Such a tool has been recently proposed in the form of a standardised short DNA sequence from an agreedupon region of the genome, which is expected to ultimately provide a means of fast and robust identification of any species on the planet: the DNA barcode. Received with as much enthusiasm by some as skepticism by others, this novel tool was set in motion on a worldwide scale by means of an international consortium of organisations (the Consortium for the Barcoding of Life), thus becoming a large-scale horizontal genomics project. While anchored within the knowledge and principles of taxonomy, DNA barcoding possesses unique characteristics which anticipate a diverse scope of new applications and benefits for society. Notably, it places the completion of the biodiversity catalogue within the reach of a single generation, with the promise to assist greatly in the discovery of new species. Alongside long-term, ultimate goals, such as democratisation of access to taxonomic knowledge and assistance in writing the encyclopaedia of life, there are several more prosaic applications that may also impact society, not only in certain scientific fields, but also in a range of social and economic activities. Here, we will use DNA barcoding of fish as an example to illustrate foreseen applications, and as a basis to stimulate reflection on potential societal impacts of this horizontal genomics project.
\end{abstract}

\section{Synopsis of the Barcode of Life Initiative}

\section{DNA barcoding: why and what for?}

The realisation of the paucity of our knowledge about the world's biodiversity, together with the limitations of current approaches to biodiversity diagnosis, are the main driving forces behind new approaches to species identification. Estimates of the number of existing eukaryotic species range from the most conservative 3.6 million up to $100+$ million, with 10 million favoured by most analysts as the nearest order of magnitude. $^{2}$ Circa 1.5 to 1.8 million species have been described to date. ${ }^{3}$ Even considering the lower estimates, we still know only a minor fraction of the immensity of life's diversity. The current rates of discovery - about 10,000 new species are described per year ${ }^{4}$ - are inadequate if such a huge gap is to be closed in the near 
future. Moreover, no more than 5\% of the named organisms are known in any biological detail. $^{5}$

Taxonomists and systematists constitute the scientific frontline for addressing issues of life's diversity. They have the central role of delineating species, naming, classifying and describing, and unravelling their phylogenies and placement in the tree of life. Ideally, these experts should make use of multiple sources of evidence and follow a hypothesis driven approach to resolve species identities and relatedness. Hence taxonomy and systematics are disciplines with high and pivotal intellectual content ${ }^{6}$ that depend on only a few taxon-specific experts.

However, intervention of experts is frequently required beyond species delineation and goes as far as routine species identification, the very basis of most research involving organisms. For those who work in other areas of science this will likely come as a surprise. In fact, learning the nuances that separate closely-allied species assemblages is so complex that few biologists, even those who have devote their careers to taxonomy, can critically discriminate more than 1000 species. This serious constraint to the diagnosis of biodiversity is exacerbated by various peculiarities of current taxonomic protocols. Many such protocols rely heavily on phenotypic characters, and frequently require lengthy and detailed inspection of the specimens, and even dissection. There is no master key that could work for different groups of taxa, or even for a single species across its different life stages. Reliable identification depends on experts who have climbed a long learning curve and who are focused on a specific group of organisms. There is also a bias of focus in particular groups such as vertebrates and insects. Taxonomic literature is often difficult to locate, and the description of a new species does not assure its future recognition. ${ }^{7}$ The compounded outcome of these difficulties, together with the shortage of taxonomic experts and resources allocated to taxonomy, impose a taxonomic impediment to understanding, utilising and conserving biological diversity. ${ }^{8,9}$ Indeed, this taxonomic impediment extends to the whole scientific community and society in general, which experience a customary difficulty to access taxonomic knowledge.

In 2003, Hebert and co-authors introduced the concept of a DNA barcode, and proposed a new approach to species identification, ${ }^{10}$ which offered great promise to counter many of the limitations above. The new approach is based on the premise that the sequence analysis of a short fragment of a single gene (eg, cytochrome c oxidase subunit 1), enables unequivocal identification of all animal species. Hence, analogously to the barcodes used in commercial products, the DNA barcode would provide a standardised tool for fast, simple, robust and precise species identification. Such a 'barcode region' would also have to evolve at a rate that would distinguish species from each other while remaining more or less identical for all members of the same species. Finally it would have to be flanked by conserved DNA regions so as to make the polymerase chain reaction (PCR), a method of targeted gene replication, practical. $^{11}$

DNA barcoding differs in many ways from conventional taxonomic identification tools and approaches, over which it offers several advantages. It permits the identification of species from fragments, and from any life-history stage, as well as 
the standardisation of a universal master key in a format that reduces ambiguity and enables direct comparison of specimens to a global reference database.

Before the introduction of DNA barcoding, various molecular methods were already applied to species identification, ${ }^{12}$ though often these were of limited scope. None had the ambition, scale and, most importantly, the degree of standardisation of barcoding. Soon, it was proposed that the DNA barcoding concept be expanded in order to embrace all eukaryotic life forms, and promised to revolutionise taxonomy and influence other allied disciplines. ${ }^{13}$ The emergence of controversy among the scientific community was not, however, unexpected. ${ }^{14}$ Some critics are concerned about known limitations of the approach (see next section), and question the ability of a single gene to provide sufficient information for such an ambitious project. ${ }^{15,16}$ Others fear that DNA barcodes will overrule conventional methods and become the unique standard for species delineation (which is different from species identification, as explained above). Or even that this fashionable and democratic tool will make species identification a frivolous, apparently straightforward task, leading to the abandonment of conventional methods, and the gradual demise of the whole scientific discipline of taxonomy and its essential intellectual input into the biological sciences. ${ }^{17,18,19}$ As explained in the next section, some of this criticism may result from misconceptions about the rationale and approach of DNA barcoding. ${ }^{20,21,22}$

\section{Rationale and approach}

Hebert and co-authors ${ }^{23}$ suggested a 650 base pair (bp) sequence of mitochondrial gene cytochrome $c$ oxidase subunit 1 (COI) as the reference DNA barcode for all animal life. This gene occurs in the mitochondria of all eukaryotic organisms, and the initial appraisal revealed consistent resolving capability at the species level for many animals. There are a few recognised limitations of this barcoding region, namely the possible lack of resolution for recently diverged species or for particular animal taxa (eg, cnidarians), or the inability to detect cases of introgressive hybridisation. These exceptions are thought to represent only a minor percentage of the target species on a global scale. Moreover, it is expected that these limitations can be tackled using additional or alternative barcoding regions in a comparatively small number of exceptional cases. Thus, while COI has been elected as the prime DNA barcode for identification of animals (and probably for macroalgae, too ${ }^{24}$ ) the pursuit of regions of the genome appropriate for use as DNA barcodes in other eukaryotic life forms (eg, plants, ${ }^{25}$ fungi ${ }^{26,27}$ ) is in progress.

The rationale and approach of DNA barcoding are essentially the same whichever region of the genome is selected. The basic premise is that for each currently known species an unequivocal match can be established with the DNA barcode obtained by reading a selected region of its genome. The DNA barcode sequence is not necessarily invariable within a species. Instead, the rationale is that individuals of a species share very similar sequences and that the barcode arrays for different species are usually distinct. This "matching hypothesis" constitutes the key starting point for launching and implementing the new bioidentification system. Every known species must be checked for the validity of this hypothesis. In doing so, a database linking a given species and respective DNA barcode array will be built. Reference barcoded

Genomics, Society and Policy, Vol.3, No.2 (2007) ISSN: 1746-5354

(C) ESRC Genomics Network. 
specimens of each species that have been identified by experts are deposited in a museum and therefore available for double-checking and for long-term study. Once this reference database is complete, it can be used to assign an unknown sample to a known species.

In comprehensive DNA barcoding studies conducted so far with Lepidoptera, ${ }^{28}$ birds, ${ }^{29}$ fish ${ }^{30}$ and crustaceans, ${ }^{31}$ a match between a DNA barcode and a known species has been found in more than $95 \%$ of the cases. Failure to obtain an unambiguous match may result from insufficient resolution of the DNA barcode (which can be the case when screening recently diverged species). However, as is the case with the studies above, ambiguities may also flag the presence of potentially unrecognised species that were overlooked by conventional methods. It is precisely in this type of setting that DNA barcoding can be of great assistance in the discovery of new species; it provides a molecular basis to test species hypotheses when data are not congruent with known species boundaries. ${ }^{32,33}$

It should be emphasised that DNA barcoding does not substitute the conventional protocol for delineating new species. ${ }^{34,35,36}$ A hypothesis-driven approach should be followed to address potential new species supported by DNA barcoding screening. Ideally, new species hypotheses should be tested against various sources of evidence (morphological, ecological, reproductive, other molecular evidence, etc.) ${ }^{37,38}$ which will continue to rely on the input of the taxonomic expert. Indeed, this novel tool will assist taxonomic experts greatly in their research efforts, and not only by releasing them from routine identifications; it also provides a fast means of screening and triage for large numbers of samples, enabling quick detection of potential new species, with consistent identification of morphologically distinct or cryptic life history stages and gender. Most importantly, the efforts of experts in the delineation and description of new species will have an immediate effect, since the new species can be readily tracked down using DNA barcoding. As new species are discovered and identifications revisited by experts, voucher specimen identifications and the global reference database can be updated and immediately effective.

\section{Organisation and framework}

In May 2004, little more than a year after the publication of Hebert and colleagues' seminal paper, ${ }^{39}$ an international consortium of organisations - the Consortium for the Barcoding of Life (CBOL) $)^{40}$ - instigated the worldwide implementation of DNA barcoding, thus launching a unique large-scale horizontal genomics project. CBOL's mission is to explore and develop the potential of DNA barcoding for research and as a practical tool for species identification. Consortium members include museums, herbaria, zoos, biodiversity research institutes, universities, conservation organisations, government agencies and private companies.

Since its inauguration, CBOL has experienced rapid development, which was particularly intense after the First Conference for the Barcoding of Life, held in February 2005 at the Natural History Museum, London. This conference constituted the first large forum for discussing DNA barcoding, and the proceedings were compiled in a special issue of Philosophical Transactions of the Royal Society. ${ }^{41,42}$ 
There was also progress in organisational aspects of CBOL with the establishment of five working groups to target specific aspects of DNA barcoding.

Currently CBOL counts more than 150 organisations from 45 countries in its membership. The first global DNA barcoding campaigns - the Fish Barcode of Life $(\text { FISH-BOL })^{43}$ and the All Birds Barcoding Initiative (ABBI) ${ }^{44}$ - have been launched, with the intention of assembling a reference database of DNA barcodes for all fish and bird species respectively. FISH-BOL expects to complete most of the inventory of all known fish species of the world by 2010 . More recently, a campaign was launched for DNA barcoding all Lepidoptera, which already exceeded 8600 species barcode records. ${ }^{45}$ Finally, a thematic international network, Barcoding of Invasive and Pest Species, ${ }^{46,47}$ is also in operation.

CBOL coordinates and promotes DNA barcoding on a worldwide scale, and endorses public access to DNA barcoding data. Both the Barcode of Life Database (BOLD) ${ }^{48}$ and existing public genomic repositories (namely the GenBank of the National Center for Biotechnology Information (NCBI), the European Molecular Biology Laboratory (EMBL) and the DNA Data Bank of Japan (DDBJ)) will provide free access to DNA barcoding data. The Barcode of Life Initiative intends also to be both integrative and integrated with other worldwide taxonomic initiatives ${ }^{49}$ such as the global Taxonomic Initiative for the Convention for Biological Diversity and the Global Biodiversity Information Facility (GBIF).

Currently DNA barcoding is a fully established approach, as recognized for example by the setting up of a 'Barcode' keyword for the identification of standard DNA barcodes in public genomic repositories ${ }^{50}$ and by the creation of a specific themesection for submission of DNA barcoding studies in the journal Molecular Ecology Notes. ${ }^{51}$ There is also 'The Barcode Blog, ${ }^{, 52}$ at Rockefeller University, which, since June 2004, has been alerting the community to new studies on barcoding.

\section{The promise of DNA barcoding}

'Imagine a world in which any person, anywhere, at any time can identify any species at little or no cost. That world is technologically upon us. ${ }^{, 53}$

By contributing to a break up of the 'taxonomic impediment', DNA barcoding promises to open doors to a diverse array of scientific and social applications and for a variety of end-users, from the scientific expert, to the individual citizens. Our improved ability to recognize existing and cryptic species will be of benefit to environmental sciences, forensics, ${ }^{54}$ pharmaceutics, agriculture, conservation, biological and molecular evolution, to countermeasures to biological warfare, to name but a few. ${ }^{55} \mathrm{We}$ describe some of the applications to fish biology and fisheries in the next section, but first we shall deal with more general impacts.

The scientific field of taxonomy itself may well be one of the most immediate beneficiaries from DNA barcoding. With this new and powerful tool, taxonomists can be freed from maintenance and routine tasks, and focus instead on the description and 
investigation of newly discovered species, ${ }^{56}$ thus greatly accelerating the rate of new entries in the encyclopaedia of life. However, crucially for addition of any species' DNA barcode to BOLD, it will remain necessary to deposit a voucher specimen, ${ }^{57}$ a requirement that emphasises the intended integration of DNA barcoding with the Linnean system.

Benefits will likely extend to more than purely technical aspects, and many view DNA barcoding as a key opportunity to revitalize the scientific discipline of taxonomy ${ }^{58}$ which has progressively become one of the most underfunded within biological sciences. ${ }^{59}$ In fact, different views on the potential impacts of DNA barcoding in taxonomy have been a source of lively debate: ${ }^{60}$ some critics suggest it will sound the death knell for a moribund but vital discipline, ${ }^{61}$ while for others it is a valuable opportunity to revolutionise and revitalise the subject. ${ }^{62,63,64}$

Such a debate might soon become a redundant one, since the prime concept and current practice of DNA barcoding is built upon establishing a match between a known vouchered species and a DNA sequence. Thus, the success of DNA barcoding is a corollary of progress in taxonomy and biodiversity inventories. The Barcode of Life Initiative has already started to draw attention to the value of taxonomy and the key role of taxonomists, and has attracted new sources of funding for the discipline. DNA barcoding has prompted unprecedented large-scale biodiversity inventories, which will provide new raw materials for taxonomy and systematics. It is raising standards for incorporating taxonomic information into genomic data repositories. ${ }^{65,66}$ Moreover, it is establishing a new and valuable type of genetic bank (by means of archiving tissue samples or DNA extracts) from which the genome of each species can be accessed in the future. ${ }^{67,68}$ Hence, benefits start to emerge, not only for taxonomy, but also for other disciplines within biological sciences and related scientific fields.

The impacts of the Barcode of Life Initiative are expected to extend beyond the scientific arena and ultimately influence society as a whole. Through improved knowledge of the planet's biodiversity, societies will be able to manage biological resources in a more sustainable and responsible manner. Ironically, the taxonomic impediment is most acute in developing countries, where biodiversity is highest. ${ }^{69}$ Features of DNA barcoding such as rapid, accurate and cost-effective specimen identification have the potential to democratize access to taxonomic information in all regions of the globe, and open the gates of biodiversity information to the ordinary non-expert citizen.

One of the most emblematic visions of the Barcode of Life Initiative is the ultimate creation of a handheld device that could be used to identify any life form anywhere and anytime at little or no cost. ${ }^{70,71}$ Such a 'Bio-pod ${ }^{\text {,72 }}$ would not only provide a species identification, but would also enable an Internet link with the corresponding entry in the encyclopaedia of life, with images and related information about that species. Below is a commentary on an article about DNA barcoding posted in a free access website. ${ }^{73}$ It synthesises in a rather spontaneous fashion the type of reaction that the ordinary citizen may have to the idea of a Bio-pod: 
This could be fantastic. If there's something in it for the end user, millions of people will be turned into field taxonomists. A known plant gives ID; a weird one means you contribute to science. Upload your location at the same time, and you have new types of data: scientists could get plant coverage. With such data useful in climate research, a person could feel good everytime they ID a plant.

Reading through Jamais' previous post, I can see this opens up a whole pandora's box of problems with patents and the openness of the whole model.

Hopefully there's a wikipedia like model for this. Are these machines available now to non-researchers? What is their cost?

This comment also captures many of the hopes for the societal benefits of the Barcode of Life Initiative, in particular the high expectations for improvements in bioliteracy. ${ }^{74}$ In this respect, DNA barcoding could become to biodiversity what the printing press was to literacy. ${ }^{75}$ A more bio-literate society as a whole would be able to take better and more responsible decisions about the management of our planet's biological heritage. The ordinary citizen would have the opportunity to become familiar with the surrounding biological diversity, and acquire a different perception of its relevance. ${ }^{76}$ It may trigger a curiosity for living organisms, and improve awareness of biodiversity threats, and the perception of how human actions can have a detrimental impact on rates of species extinctions and ecosystem change. Eventually, a more bio-literate society could produce 'greener' individuals, who are more environmentally-responsible in their daily actions, and willing to undertake pro-active measures to minimize their own impact on the planet's biodiversity.

\section{The example of fish DNA barcoding}

Fish provide a suitable model for testing the implementation of DNA barcoding at a worldwide scale. Although they constitute the largest vertebrate group (about $50 \%$ of all vertebrate species), they have a manageable number of species: c.20,000 marine species (15,648 in Fish Base; 91 with subspecies); c.15,000 freshwater species (13,544 in Fish Base; 152 with subspecies) (705 species occur in both marine and freshwater systems); and c. 80 brackish species (82 in Fish Base; 1 with subspecies). They are very diverse systematically, comprising three major groups of organisms: the jawless fish (Superclass Agnatha), such as lampreys hagfish; the cartilaginous fish (Class Chondrichthyes), including sharks and rays; and the immense variety of the bony fish (Superclass Osteichthyes) which include lungfishes, eels, tunas, sea horses, etc. $^{77}$

Fish are also of economic value as a food source. Global figures for the value at first sale in 2000, is circa US\$81 billion for capture fisheries and about US\$52 billion for aquaculture (excluding plants). ${ }^{78}$ In the same year the estimate for retail trade for ornamental fish in the USA alone was US\$3 billion, and in 1984 in Australia the value of recreational sports fishing was estimated in US\$2 billion.

Fish and fisheries resources comprise a key target group from which it is anticipated that DNA barcoding will bring larger and more immediate benefits. ${ }^{79}$ Such a system 
will offer a simple - and increasingly rapid and inexpensive - means of unambiguously identifying not only whole fish, but fish eggs and larvae, fish fragments, fish fillets and processed fish. This capability will yield more rigorous and extensive data on recruitment, ecology and geographic ranges of fisheries resources, and improved knowledge of nursery areas and spawning grounds, with evident impacts at the fisheries management and conservation levels. For example, the possibility of rigorous identification of fish species from eggs and larvae could be particularly fruitful, since phenotypic identification of early life stages can be especially difficult. ${ }^{80}$ A study testing the application of molecular techniques in species identification of fish eggs revealed that over $60 \%$ of the eggs were misidentified when phenotypic characters were used. ${ }^{81}$ Eggs from haddock and whiting may have been reported as cod's eggs in previous surveys, possibly leading to an inflation of stock assessments of cod in the Irish Sea. Moreover, early stage haddock eggs were detected in the Irish Sea, indicating the presence of a spawning stock of this species previously unknown to that region. ${ }^{82}$ In a context of environmental change, induced, for instance, by global warming, the ability to rigorously identify fish species at all life history stages from egg to adult is particularly useful to assess changes in geographic distribution ranges, spawning grounds and nursery areas.

Another valuable application envisaged for DNA barcoding is the identification of prey-remains from predators' stomach contents. This could provide more detailed information about aquatic trophic chains, revealing which fish species are preyed upon by other fish species ${ }^{83}$ or seabirds. ${ }^{84}$ This type of information could then be incorporated into ecological models and provide new data for use in management and conservation.

Potential forensic applications of fish DNA barcoding include the monitoring of fisheries quotas and by-catch, inspection of fisheries markets and products, the control of trade in endangered species, and improvements in the traceability of fish products. In Australian waters, for example, sharks are illegally captured, largely for their fins alone. Quality sharks' fins can sell for \$6,000-\$8,000/ $\mathrm{kg}$ in Hong Kong, and it is estimated that globally more than 100 million sharks are killed every year. Sharks are a particularly susceptible animal, since they are slow growing, long lived, undergo a long gestation and have low fecundity. Many species are morphologically very similar, and many are protected. ${ }^{85} \mathrm{~A}$ tool enabling precise identification of shark species from fins, from the fisheries boat to the soup in the restaurant, could be of great utility for law enforcement and conservation of endangered species. ${ }^{86}$ Such a tool could also be used for detection of fraudulent species substitutions in fish markets and fish food products, a practice that is generating concern among consumers. ${ }^{87} \mathrm{~A}$ striking example comes from the Red Snapper (Lutjanus campechanus), which is one of the most economically important fisheries in the Gulf of Mexico, and which has been subject to stringent fishing restrictions due to stock depletion. Marko and colleagues $^{88}$ used sequences of the mtDNA gene cytochrome b, in an approach very similar to DNA barcoding, to show that as much as $77 \%$ of the L. campechanus fillets were mislabelled in USA markets. This level of mislabelling may adversely affect estimates of stock size and contribute to the false impression among consumers that the supply of fish is keeping up with demand. 
In this section we have illustrated several potential and actual applications of fish DNA barcoding, which can have direct impacts on various activities from fisheries management to traceability of products in the food supply chain. These are in addition to the scientific applications mentioned in previous sections, such as detection and tracking of undescribed species, clarification of taxonomic uncertainties (eg, cryptic species) and identification of historical, archived and museum material. ${ }^{89}$

\section{Conclusions}

Humankind's outstanding technological and scientific achievements during the late $20^{\text {th }}$ century include space exploration, the unravelling of the human genome, and the cloning of mammals. In the face of such accomplishments, the paucity of our knowledge of the world's biodiversity is both puzzling and disappointing.

Tackling the inventory of the planet's biodiversity is in itself a colossal task. The Barcode of Life Initiative promises to accomplish that task in the timescale of a single generation. Only time will tell if it succeeds. Like the Human Genome Project, DNA barcoding is not free of controversy. While in the aftermath of the Human Genome Project it became evident that information per se does not generate knowledge, there is today broad recognition of the value and relevance of that project, to the extent that various genome projects of other organisms have followed.

Among other virtues, DNA barcoding has already focused attention on problems of biodiversity. There is little doubt of the worth of the numerous applications of the technology, as for example described in the context of fish and fisheries. The success of various wider and more ambitious historical, philosophical, and sociological goals of barcoding will depend initially on the approach of the scientific community, but also on current and future recognition, investment and support from society.

Perhaps the decisive test for DNA barcoding will be the ability to effectively convert the immense information to be collected into tangible scientific knowledge - the completion of the encyclopaedia of life. Accomplishing this task will improve citizen bio-literacy of the world's biodiversity, and possibly engender a new vision and attitude towards non-human life-forms and their conservation and sustainable utilisation. The role of the classical amateur naturalist so typical of the Victorian era, would become extended to those with limited taxonomic knowledge, although taxonomic expertise would still underpin all barcoding applications. Should DNA barcoding succeed in its mission, the concurrent progress in taxonomy may rank among the most important scientific legacies of the early $21^{\text {st }}$ century.

\footnotetext{
${ }^{1}$ The authors are at the School of Biological Sciences, University of Wales, Bangor, UK. Correspondence to f.o.costa@bangor.ac.uk.

${ }^{2}$ E.O. Wilson. Taxonomy as a fundamental discipline. Philosophical Transactions of the Royal Society of London Series B 2004; 359 (1444): 739.

${ }^{3}$ Wilson op. cit. note 2
} 
${ }^{4}$ R.M. May. Tomorrow's taxonomy: collecting new species in the field will remain the rate-limiting step. Philosophical Transactions of the Royal Society of London Series B 2004; 359 (1444): 733-734.

${ }^{5}$ P.H. Raven. Taxonomy: Where are we now? Philosophical Transactions of the Royal Society of London Series B 2004; 359 (1444): 729-730.

${ }^{6}$ Wilson op.cit. note 2.

${ }^{7}$ K.J. Gaston and M.A. O'Neill. Automated species identification: why not? Philosophical Transactions of the Royal Society of London Series B 2004; 359 (1444): 655-667.

${ }^{8}$ J.E. Rodman and J.H. Cody. The taxonomic impediment overcome: NSF's partnerships for enhancing expertise in taxonomy (PEET) as a model. Systematic Biology 2003; 52 (3): 428-435.

${ }^{9}$ Q.D. Wheeler, P.H. Raven and E.O. Wilson. Taxonomy: Impediment or expedient? Science 2004; 303 (5656): 285.

${ }^{10}$ P.D.N. Hebert et al. Biological identifications through DNA barcodes. Proceedings of the Royal Society of London Series B 2003; 270 (1512): 313-321.

${ }^{11}$ D.E. Schindel and S.E. Miller. DNA barcoding and the Consortium for the Barcode of Life. In: Success stories in the implementation of the programmes of work on dry and sub-humid lands and the Global Taxonomy Initiative. Convention on Biological Diversity Technical Series 2005; 21: 144-146 (available at http://www.cbd.int/doc/publications/cbd-ts-21.pdf).

12 J.C. Avise. 2004. Molecular Markers, Natural History, and Evolution. 2nd edition. Massachussets, Sinauer.

${ }^{13}$ Hebert, P.D.N. and T.R. Gregory. The promise of DNA barcoding for taxonomy. Systematic Biology 2005; 54 (5): 852-859.

${ }^{14}$ V.S. Smith. DNA barcoding: Perspectives from a "Partnerships for Enhancing Expertise in Taxonomy" (PEET) debate. Systematic Biology 2005; 54 (5): 841-844.

${ }^{15}$ C. Moritz and C. Cicero. DNA barcoding: Promise and pitfalls. PLoS Biology 2004; 2 (10): 15291531.

${ }^{16}$ D. Rubinoff, S. Cameron and K. Will. Are plant DNA barcodes a search for the Holy Grail? Trends in Ecology \& Evolution 2006; 21(1): 1-2.

${ }^{17}$ M.C. Ebach and C. Holdrege. More taxonomy, not DNA barcoding. Bioscience 2005; 55 (10): 822823.

${ }^{18}$ M.R.de Carvalho et al. Revisiting the taxonomic impediment. Science 2005; 307 (5708): 353-353.

${ }^{19}$ M.C. Ebach and C. Holdrege. DNA barcoding is no substitute for taxonomy. Nature 2005; 434 (7034): 697

${ }^{20}$ Hebert and Gregory, op.cit. note 13.

${ }^{21}$ R. DeSalle. Species discovery versus species identification in DNA barcoding efforts: Response to Rubinoff. Conservation Biology 2006; 20 (5): 1545-1547.

${ }^{22}$ D. Rubinoff. DNA barcoding evolves into the familiar. Conservation Biology 2006; 20 (5): 15481549 .

${ }^{23}$ Hebert et al. op. cit. note 10.

${ }^{24}$ G.W. Saunders. Applying DNA barcoding to red macroalgae: a preliminary appraisal holds promise for future applications. Philosophical Transactions of the Royal Society B 2005; 360 (1462): 18791888.

${ }^{25}$ R.S. Cowan et al. 300,000 species to identify: problems, progress, and prospects in DNA barcoding of land plants. Taxon 2006; 55 (3): 611-616.

${ }^{26}$ X.J.I.A. Min and D.H. Hichey. Assessing the effect of varying sequence length on DNA barcoding of fungi. Molecular Ecology Notes 2007; 7 (3): 365-373.

${ }^{27}$ S. Yang. Researchers barcode DNA of 6,000 fungi species in Venice museum. UCBerkeley News. 2006 (available at http://www.berkeley.edu/news/media/releases/2006/12/13 fungi.shtml).

${ }^{28}$ M. Hajibabaei et al. DNA barcodes distinguish species of tropical Lepidoptera. Proceedings of the National Academy of Sciences 2006. 103 (4): 968-971.

${ }^{29}$ P.D.N. Hebert et al. Identification of birds through DNA barcodes. PLoS Biology 2004; 2 (10): 1657-1663.

${ }^{30}$ R.D.Ward et al. DNA barcoding Australia's fish species. Philosophical Transactions of the Royal Society Series B 2005; 360 (1462): 1847-1857.

${ }^{31}$ F.O. Costa et al. Biological identifications through DNA barcodes: the case of the Crustacea.

Canadian Journal of Fisheries and Aquatic Sciences 2007; 67 (2): 272-295. 
${ }^{32}$ P.D.N. Hebert et al. Ten species in one: DNA barcoding reveals cryptic species in the neotropical skipper butterfly Astraptes fulgerator. Proceedings of the National Academy of Sciences 2004; 101 (41): 14812-14817.

${ }^{33}$ A. Gomez et al. Mating trials validate the use of DNA barcoding to reveal cryptic speciation of a marine bryozoan taxon. Proceedings of the Royal Society Series B- 2007; 274 (1607): 199-207.

${ }^{34}$ DeSalle, op. cit. note 21.

${ }^{35}$ Wheeler et al, op. cit. note 9.

${ }^{36}$ M. Hajibabaei, G.A.C. Singer and D.A. Hickey. Benchmarking DNA barcodes: an assessment using available primate sequences. Genome 2006; 49 (7): 851-854.

${ }^{37}$ DeSalle, op. cit. note 21.

${ }^{38}$ A.P. Vogler and M.T. Monaghan. Recent advances in DNA taxonomy. Journal of Zoological Systematics and Evolutionary Research 2006; 45: 1-10.

${ }^{39}$ Hebert et al., op. cit. note 10 .

${ }^{40} \mathrm{CBOL}$ homepage: $\mathrm{http} / /$ barcoding.si.edu/

${ }^{41}$ V. Savolainen et al. DNA Barcoding of Life. Themed issue of the Philosophical Transactions of the Royal Society B 2005; 360: 1803-1980.

${ }^{42}$ Reviewed in H.C.J. Godfray. To boldly sequence. Trends in Ecology \& Evolution 2006; 21: 603604.

${ }^{43}$ Fish-BOL homepage: http://www.fishbol.org

${ }^{44}$ ABBI homepage: http://www.barcodingbirds.org/

${ }^{45}$ All Leps homepage: http://www.lepbarcoding.org/

${ }^{46}$ K.F. Armstrong and S.L. Ball. DNA barcodes for biosecurity: invasive species identification. Philosophical Transactions of the Royal Society Series B 2005; 360 (1462): 1813-1823.

${ }^{47}$ S.L. Ball, K.F. Armstrong and G. Roderick. INBIPS: The International Network for the Barcoding of Invasive and Pest Species. In: Success stories in the implementation of the programmes of work on dry and sub-humid lands and the Global Taxonomy Initiative. Convention on Biological Diversity

Technical Series 2005; 21: 160-162 (available at http://www.cbd.int/doc/publications/cbd-ts-21.pdf).

${ }^{48}$ S. Ratnasingham and P.D.N. Hebert. BOLD: The Barcode of Life Data System (http://www.barcodinglife.org). Molecular Ecology Notes 2007; 7 (3): 355-364.

${ }^{49}$ DeSalle, op. cit. note 21 . Discusses the value of DNA barcoding to facilitate integration and interaction of current large scale biodiversity databases initiatives.

${ }^{50} \mathrm{R}$. Hanner et al. Data standards for DNA barcode records: indexing an encyclopaedia of life. Submitted, 2007. Currently available as: R. Hanner. Proposed standards for barcode records in INSDC (BRIs), 2005; http://barcoding.si.edu/protocols.html.

${ }^{51} \mathrm{http}: / /$ www.blackwellpublishing.com/journal.asp? $\mathrm{ref}=1471-8278 \&$ site $=24$

$52 \mathrm{http}: / /$ phe.rockefeller.edu/barcode/blog/

${ }_{53}$ Report of the Workshop 'Taxonomy, DNA and the Barcode of Life'. Banbury Center, Cold Spring Harbor Laboratory, NY September 10-12, 2003.

${ }^{54} \mathrm{~N}$. Dawnay et al. Validation of the Barcoding gene COI for use in forensic genetic species identification. Forensic Science International 2006; (in press).

${ }^{55} \mathrm{~V}$. Savolainen et al. Towards writing the encyclopaedia of life: an introduction to DNA barcoding. Philosophical Transactions of the Royal Society Series B 2005; 360 (1462): 1805-1811.

${ }^{56}$ Ibid.

${ }^{57}$ C. Schander and E. Willassen. What can biological barcoding do for marine biology? Marine Biology Research, 2005; 1 (1): 79-83. Underlines the need to link DNA barcodes with museum vouchers.

${ }^{58}$ CBOL, op. cit. note 40 .

${ }^{59}$ Wilson, op.cit. note 2.

${ }^{60}$ Smith, op. cit. note 14 .

${ }^{61}$ Will, K.W., B.D. Mishler, and Q.D. Wheeler, The perils of DNA barcoding and the need for integrative taxonomy. Systematic Biology, 2005. 54 (5): 844-851.

${ }^{62}$ Hebert and Gregory, op. cit. note 13.

${ }^{63}$ D.E. Schindel and S.E. Miller. DNA barcoding a useful tool for taxonomists. Nature 2005; 435 (7038): 17.

${ }^{64}$ T.R. Gregory. DNA barcoding does not compete with taxonomy. Nature 2005; 434 (7037): 1067.

${ }^{65}$ Hanner et al, op. cit. note 50 . 
${ }^{66}$ R.H. Nilsson et al. Taxonomic reliability of DNA sequences in public sequence databases: a fungal perspective. PLoS One, 2006. 1: (e59). This paper raises the issue of the taxonomic reliability of DNA sequences in public sequence databases in a fungal context, but in fact this is currently a concern for all taxa. The strict conditions demanded for DNA barcoding records are helping to raise the bar for taxonomic reliability of sequences deposited in public databases.

${ }^{67}$ The Ocean Genome Legacy: http://www.oglf.org/

${ }^{68}$ P.R. Becker et al., Environmental specimen banking. Journal of Environmental Monitoring 2006; 8 (8): 776-778. For discussion on the importance of environmental specimen banking for different uses, including access to the genomes.

${ }^{69}$ Schindel and Miller, op. cit. note 11.

${ }^{70}$ Savolainen et al, op. cit. note 55.

${ }^{71}$ D.H. Janzen. Now is the time. Philosophical Transactions of the Royal Society of London Series B 2004; 359 (1444): 731-732.

${ }^{72}$ The expression 'Bio-pod' is taken from http://www.flmnh.ufl.edu/cowries/barcoding intro.pdf.

${ }^{73}$ Posted by: Daniel Haran at October 20, 2005 05:37 PM at http://www.worldchanging.com/archives/003655.html.

${ }^{74}$ Janzen, op. cit. note 71.

${ }^{75}$ M. Holloway. Democratizing taxonomy. Conservation in Practice 2006; 7: 14-21.

${ }^{76}$ Savolainen et al, op. cit. note 55 .

${ }^{77}$ R.D. Ward et al. DNA barcoding Australia's fish species. Philosophical Transactions of the Royal Society B 2005; 360 (1462): 1847-1857.

${ }^{78}$ FAO. The state of world fisheries and aquaculture, part 1: world review of fisheries and aquaculture. 2002, Rome: Food and Agricultural Organization, Fisheries Department.

${ }^{79}$ J. Lleonart, M. Taconet and M. Lamboeuf. Integrating information on marine species identification for fishery purposes. Marine Ecology-Progress Series 2006; 316: 231-238. Underlines the challenges of species identification of marine fisheries and the need for a global database network to share taxonomic knowledge. DNA barcoding would be of great assistance to such an approach.

${ }^{80}$ G.G. Pegg et al. MtDNA barcode identification of fish larvae in the southern Great Barrier Reef, Australia. Scientia Marina 2006; 70: 7-12.

${ }^{81}$ C.J. Fox et al. TaqMan DNA technology confirms likely overestimation of cod (Gadus morhua L.) egg abundance in the Irish Sea: implications for the assessment of the cod stock and mapping of spawning areas using egg-based methods. Molecular Ecology 2005; 14 (3): 879-884.

82 Ibid.

${ }^{83}$ M.F. Sigler, et al. Diet of Pacific sleeper shark, a potential Steller sea lion predator, in the north-east Pacific Ocean. Journal of Fish Biology 2006; 69 (2): 392-405. Molecular techniques were used to identify some of the prey in stomach contents of the Pacific sleeper shark.

${ }^{84}$ R.A. Phillips et al. Diet of the northern fulmar Fulmarus glacialis: reliance on commercial fisheries? Marine Biology, 1999; 135 (1): 159-170. Availability of a DNA barcodes library of fish would have helped identify prey species of the northern fulmar.

${ }^{85}$ R. D. Ward, pers. comm.

${ }^{86}$ R.W.K. Chan, et al. Application of DNA-based techniques for the identification of whaler sharks (Carcharhinus spp.) caught in protective beach meshing and by recreational fisheries off the coast of New South Wales. Fishery Bulletin 2003; 101 (4): 910-914.

${ }^{87}$ M. Trotta et al. Multiplex PCR method for use in real-time PCR for identification of fish fillets from grouper (Epinephelus and Mycteroperca species) and common substitute species. Journal of Agricultural and Food Chemistry 2005; 53 (6): 2039-2045.

${ }^{88}$ P.B. Marko et al. Mislabelling of a depleted reef fish. Nature 2004; 430 (6997): 309-310.

${ }^{89}$ M. Hajibabaei et al. A minimalist barcode can identify a specimen whose DNA is degraded. Molecular Ecology Notes 2006; 6 (4): 959-964. 